\title{
RECIPROCAL RELATIONS IN IRREVERSIBLE PROCESSES. I.
}

\author{
By LaRs ONSAGER \\ Department of Chemistry, Brown University
}

(Received December 8, 1930)

\begin{abstract}
Examples of coupled irreversible processes like the thermoelectric phenomena, the transference phenomena in electrolytes and heat conduction in an anisotropic medium are considered. For certain cases of such interaction reciprocal relations have been deduced by earlier writers, e.g., Thomson's theory of thermoelectric phenomena and Helmholtz' theory for the e.m.f. of electrolytic cells with liquid junction. These earlier derivations may be classed as quasi-thermodynamic; in fact, Thomson himself pointed out that his argument was incomplete, and that his relation ought to be established on an experimental basis. A general class of such relations will be derived by a new theoretical treatment from the principle of microscopic reversibility. ( $\$ \$ 1-2$.) The analogy with a chemical monomolecular triangle reaction is discussed; in this case a a simple kinetic consideration assuming microscopic reversibility yields a reciprocal relation that is not necessary for fulfilling the requirements of thermodynamics ( $\$ 3$ ). Reciprocal relations for heat conduction in an anisotropic medium are derived from the assumption of microscopic reversibility, applied to fluctuations. ( $\$ 4$.) The reciprocal relations can be expressed in terms of a potential, the dissipation-function. Lord Rayleigh's "principle of the least dissipation of energy" is generalized to include the case of anisotropic heat conduction. A further generalization is announced. ( $\$ 5$.) The conditions for stationary flow are formulated; the connection with earlier quasithermodynamic theories is discussed. ( $\$ 6$.$) The principle of dynamical reversibility$ does not apply when (external) magnetic fields or Coriolis forces are present, and the reciprocal relations break down. ( $\$ 7$.
\end{abstract}

\section{INTRODUCTION}

W

HEN two or more irreversible transport processes (heat conduction, electrical conduction and diffusion) take place simultaneously in a thermodynamic system the processes may interfere with each other. Thus an electric current in a circuit that consists of different metallic conductors will in general cause evolution or absorption of heat at the junctions (Peltier effect). Conversely, if the junctions are maintained at different temperatures an electromotive force will usually appear in the circuit, the thermoelectric force: the flow of heat has a tendency to carry the electricity along.

In such cases one may naturally suspect reciprocal relations by analogy to the reciprocal relations which connect forces and displacements in the equilibrium theory of mechanics and in thermodynamics. Relations of this type have been proposed and discussed by many writers. The earliest of them all is due to $\mathrm{W}$. Thomson $;^{1}$ it deals with thermoelectric phenomena. We shall cite Thomson's reciprocal relation in a simple form as a symmetry condition for the relations which connect the forces with the velocities. The electric current we shall call $J_{1}$, the heat flow $J_{2}$. The current is driven by the elec-

${ }^{1}$ W. Thomson (Lord Kelvin), Proc. Roy. Soc. Edinburgh 1854, p. 123; Collected Papers I, pp. $237-41$. 
tromotive force, which we shall call $X_{1}$. In corresponding units the "force" which drives the flow of heat will be:

$$
X_{2}=-\frac{1}{T} \operatorname{grad} T,
$$

where $T$ denotes the absolute temperature (Carnot). If the heat flow and the current were completely independent we should have relations of the type:

$$
\begin{aligned}
& X_{1}=R_{1} J_{1} \\
& X_{2}=R_{2} J_{2}
\end{aligned}
$$

where $R_{1}$ is the electrical resistance and $R_{2}$ a "heat resistance." However, since the two processes interfere with each other we must use the more complicated phenomenological relations

$$
\begin{aligned}
& X_{1}=R_{11} J_{1}+R_{12} J_{2} \\
& X_{2}=R_{21} J_{1}+R_{22} J_{2} .
\end{aligned}
$$

Here Thomson's contention is :

$$
R_{12}=R_{21} .
$$

Thomson arrived at this relation from thermodynamic reasoning, but he had to make one additional assumption, namely: "The electromotive forces produced by inequalities of temperature in a circuit of different metals, and the thermal effects of electric current circulating in it, are subject to the laws which would follow from the general principles of the thermodynamic theory of heat if there were no conduction of heat from one part of the circuit to another." Thomson thought this assumption very plausible. Even so, he cautiously considered his reciprocal relation (1.2) a conjecture, to be confirmed or refuted by experiment, since it could not be derived entirely from fundamental principles known at that time. At present Thomson's relation is generally accepted, because it has been confirmed within the limits of error of the best measurements. As regards the theory, the same relation has frequently been found as a by-product of investigations in the electron theory of metals. However, Thomson's relation has not been derived entirely from recognized fundamental principles, nor is it known exactly which general laws of molecular mechanics might be responsible for the success of Thomson's peculiar hypothesis.

In the following, a general class of reciprocal relations in irreversible processes will be derived from the assumption of microscopic reversibility. No further assumptions will be necessary, except certain theorems borrowed from the general theory of fluctuations. Among the relations to be derived, many have been proposed before, but some will be new. An important group among these relations can be summarized in a variation-principle, which is nothing but an extension of Lord Rayleigh's "principle of the least dissipation of energy"; we shall retain the name for the extended principle. According to this theorem the rate of increase of the entropy plays the rôle of a potential. 
Thomson's hypothesis covers only part of the cases which we are going to consider, yielding the same results as the more general "principle of the least dissipation of energy." This connection will be discussed in $\S 6$; here, however, we shall comment on the natural interpretation of Thomson's hypothesis, because his formulation is somewhat ambiguous: He assumes that the temperature differences present are bound to cause a certain degradation of energy by conduction of heat; if another irreversible process (electrical conduction) takes place simultaneously, this process must cause an additional degradation of energy, making the total rate of increase of the entropy greater than it would be by heat conduction alone.

The derivation of such results from the principle of microscopic reversibility will hardly be a surprise to workers who are acquainted with the theory of irreversible processes. In many familiar cases this principle guarantees an independent balancing of different classes of molecular processes maintaining a statistical equilibrium. The writer actually conceived the idea for the new derivation of reciprocal relations by comparing the relations due to Thomson, ${ }^{1}$ Helmholtz ${ }^{2}$ and others with the conditions for reversibility in certain chemical reactions, as presented in $\$ 3$.

The principle of microscopic reversibility is less general than the fundamental laws of thermodynamics; the consequent limitations to our reciprocal relations will be briefly described in $\S 7$. It is easy to show that Thomson's relation is no thermodynamic necessity. According to (1.2) the rate of production of entropy per unit volume of the conductor equals

$$
\frac{d S}{d t}=\frac{1}{T}\left(X_{1} J_{1}+X_{2} J_{2}\right)=\frac{1}{T}\left(R_{11} J_{1}^{2}+\left(R_{12}+R_{21}\right) J_{1} J_{2}+R_{22} J_{2}^{2}\right) .
$$

Thermodynamics requires only:

$$
d S / d T>0
$$

identically, except when $J_{1}$ and $J_{2}$ vanish simultaneously, or, by simple algebra:

$$
R_{12}+R_{21}<2\left(R_{11} R_{22}\right)^{1 / 2} .
$$

This condition (in a somewhat different form) was given by Boltzmann. ${ }^{3}$

In the present communication only one special case, namely heat conduction in an anisotropic medium (crystal), will be adequately treated $(\$ 4)$. This limitation allows a simplified derivation, which nevertheless brings out clearly the essential ideas of the new theoretical treatment. The derivation of other reciprocal relations, including Thomson's, will be reserved for later publication; here we shall merely enumerate the most important cases.

\section{Examples of Mutual Interaction of Irreversible Processes}

The formulation (1.1) of the laws of irreversible processes in terms of resistances $R_{11}$, etc., is well adapted to a comparison with the thermodynamic requirements; but as a rule it is a little easier to see the physical meaning of such

${ }^{2}$ H. v. Helmholtz, Wied. Ann. 3, 201 (1876); Wiss. Abh. 1, 840.

${ }^{3}$ L. Boltzmann, Wien. Ber. 96, 1258 (1887). 
laws when they are expressed in terms of conductances $L_{11}, L_{12} \cdots$ etc.:

where:

$$
\begin{aligned}
& J_{1}=L_{11} X_{1}+L_{12} X_{2} \\
& J_{2}=L_{21} X_{1}+L_{22} X_{2}
\end{aligned}
$$

$$
L_{11}=R_{22} /\left(R_{11} R_{22}-R_{12} R_{21}\right)
$$

etc. The reciprocal relation (1.2) takes the form:

$$
L_{12}=L_{21} \text {. }
$$

So far we have only mentioned the interaction of heat conduction and electrical conduction. In mixtures of gases and in solutions a third transport process is possible, namely diffusion. Experience shows that any two possible transport processes are likely to interfere with each other to some extent.

It is well known that an electric current in a conductor of the second kind causes transport of matter. Conversely, a concentration gradient between two identical reversible electrodes causes an electromotive force. The relations between "forces" and velocities may again be expressed in the form (2.1). $J_{1}$ may again be the electric current, and $X_{1}$ the e.m.f.; for $J_{2}$ we take the flow of solute relative to the solvent, for $X_{2}$ the gradient $-\operatorname{grad} \mu=X_{2}$ of the thermodynamic potential $\mu$ of the solute. The coefficients $L_{11} \cdots L_{22}$ are connected, in a manner which we need not discuss in detail, with the electrical resistance, the diffusion coefficient, the "transference number" and the e.m.f. caused by a given concentration gradient. Usually the fourth is not mentioned, since Helmholtz ${ }^{3}$ has derived the reciprocal relation.

$$
L_{12}=L_{21} \text {. }
$$

His derivation is quite analogous to Thomson's treatment of the thermoelectric phenomena, and it suffers from the same weakness. However, the experiments confirm the result, Nernst ${ }^{4}$ has given a kinetic derivation from assumptions that are somewhat specialized, and the theorem is generally accepted.

For the interaction between heat conduction and diffusion a reciprocal theorem has been derived by Eastman $;^{5}$ the case is quite analogous to the preceding. The diffusion caused by a temperature gradient is known as the Soret effect; the inverse effect has been demonstrated in a qualitative manner for a mixture of gases. ${ }^{6}$ Eastman has also discussed thermoelectric forces in electrolytes. In that case three different transport processes are involved simultaneously. The phenomenological relations can be expressed in the form

$$
\begin{aligned}
& J_{1}=L_{11} X_{1}+L_{12} X_{2}+L_{13} X_{3} \\
& J_{2}=L_{21} X_{1}+L_{22} X_{2}+L_{23} X_{3} \\
& J_{3}=L_{31} X_{1}+L_{32} X_{2}+L_{33} X_{3}
\end{aligned}
$$

and we may suspect $3 \cdot 2 / 2=3$ reciprocal relations

$$
L_{12}=L_{21} ; L_{13}=L_{31} ; L_{23}=L_{32} \text {. }
$$

${ }^{4}$ W. Nernst, Zeits. f. physik. Chem. 2, 613 (1888).

${ }^{5}$ E. D. Eastman, J. Am. Chem. Soc. 48, 1482 (1926); 50, 283, 292 (1928).

6 Dufour, Arch. d. sc. phys. et nat. Genf 45, 9 (1872); Pogg. Ann. 148, 490 (1873). 
The case of simultaneous diffusion of several substances in the same solution completes the list of possibilities for coupling between transport processes in isotropic bodies.

Transport processes in anisotropic bodies afford a few interesting examples of mutual interaction, for instance the conduction of heat in crystals of low symmetry. In the most general case of a triclinic crystal the phenomenological relations can be written in the form (2.3). We chose a cartesian frame of coordinates $x_{1}, x_{2}, x_{3}$; then $J_{1}, J_{2}, J_{3}$ are the components of the heat flow along these axes, and the "forces" are $X_{1}=-(1 / T) \partial T / d x_{1}$ etc. In a suitable frame of reference $x_{1}{ }^{*}, x_{2}{ }^{*}, x_{3}{ }^{*}$ parallel to the main axes of the ellipsoid:

$L_{11} x_{1}^{2}+\left(L_{12}+L_{21}\right) x_{1} x_{2}+\cdots+L_{33} x_{3}{ }^{2}=\lambda_{1} x_{1}{ }^{* 2}+\lambda_{2} x_{2}{ }^{* 2}+\lambda_{3} x_{3}{ }^{* 2}=$ const.

the equations of heat conduction take the form

$$
\begin{aligned}
& J_{1}{ }^{*}=\lambda_{1} X_{1}{ }^{*}+\omega_{3} X_{2}{ }^{*}-\omega_{2} X_{3}{ }^{*} \\
& J_{2}{ }^{*}=-\omega_{3} X_{1}{ }^{*}+\lambda_{2} X_{2}{ }^{*}+\omega_{1} X_{3}{ }^{*} \\
& J_{3}{ }^{*}=\omega_{2} X_{1}{ }^{*}-\omega_{1} X_{2}{ }^{*}+\lambda_{3} X_{3}{ }^{*} .
\end{aligned}
$$

If the reciprocal relations (2.4) are valid, then:

$$
\omega_{1}=\omega_{2}=\omega_{3}=0
$$

and the conducting properties of the crystal are entirely determined by the ellipsoid (2.5); whenever the gradient of the temperature is parallel to one of the axes $x^{*}$ the heat flows in exactly the same direction. If $\omega_{1}, \omega_{2}, \omega_{3}$ do not vanish there may still be three such directions of direct heat flow, in which case these directions will no longer be perpendicular to each other, or there may be only one such direction. For instance, for crystals belonging to the tetragonal or hexagonal systems the inherent symmetry fixes the axes $x_{1}{ }^{*}=x_{1}$; $x_{2}{ }^{*}=x_{2} ; x_{3}{ }^{*}=x_{3}$ and demands certain relations between the coefficients, so that (2.3) and (2.6) take the form

$$
\begin{aligned}
& J_{1}=\lambda_{1} X_{1}+\omega_{3} X_{2} \\
& J_{2}=-\omega_{3} X_{1}+\lambda_{1} X_{2} \\
& J_{3}=\lambda_{3} X_{3} .
\end{aligned}
$$

If clockwise and counterclockwise rotations around the axis $x_{3}$ are equivalent $\omega_{3}$ vanishes for reasons of symmetry, but certain classes of the crystallographic systems in question do not possess such a symmetry. When $\omega_{3}$ does not vanish the flow of heat in the $x_{1}, x_{2}$ plane will always form an angle:

$$
\arctan \left(-\omega_{3} / \lambda_{1}\right)
$$

with the temperature gradient. If we should take a circular plate of such a crystal, cut parallel to the base $\left(x_{1}, x_{2}\right.$ plane), and heat it in the middle, maintaining cylindrical symmetry, the heat would flow in spirals. Attempts by Soret and W. Voigt to detect this spiral motion met with negative results, in spite of a very sensitive method. ${ }^{7}$

${ }^{7}$ Ch. Soret, Arch. de Geneve 29, no. 4 (1893); 32, no. 12 (1894). W. Voigt, Gött. Nachr. 87, (1903). 
The case of electrical conduction in highly anisotropic crystals is quite analogous to the heat conduction, and the thermoelectric phenomena in crystals afford a variety of possibilitities for reciprocal relations. We need only mention these two examples; a detailed discussion would not bring out any new features of the general problem.

\section{Analogy with Chemical Reactions}

We shall compare (2.3) with the equations for a chemical monomolecular triangle reaction. Suppose that a certain substance may exist in a homogeneous phase in three different forms $A, B, C$. Suppose further that any one of these may spontaneously transform itself directly into either of the others according to the scheme

$$
\underset{C \Downarrow}{A \bowtie}
$$

We shall assume that the reactions obey a simple mass-action law. That is, the fraction of $A$ molecules which will change into $B$ in a given short time $\Delta t$ is

$$
k_{B A} \Delta t
$$

where $k_{B A}$ is a constant. Then the rates of change of the amounts $n_{A}, n_{B}, n_{C}$ are given by the equations

$$
\begin{aligned}
& \frac{d n_{A}}{d t}=-\left(k_{B A}+k_{C A}\right) n_{A}+k_{A B} n_{B}+k_{A C} n_{C} \\
& \frac{d n_{B}}{d t}=k_{B A} n_{A}-\left(k_{A B}+k_{C B}\right) n_{B}+k_{B C} n_{C} \\
& \frac{d n_{C}}{d t}=k_{C A} n_{A}+k_{C B} n_{B}-\left(k_{A C}+k_{B C}\right) n_{C} .
\end{aligned}
$$

If one or several of the coefficients $k_{B A}$ etc. vanish the case becomes trivial; we shall therefore assume that they are all $\gtrless 0$. This condition is by itself sufficient to insure finite equilibrium concentrations $\bar{n}_{A}, \bar{n}_{B}, \bar{n}_{C}$, which are given by the relations

$$
\frac{d \bar{n}_{A}}{d t}=0=-\left(k_{B A}+k_{C A}\right) \bar{n}_{A}+k_{A B} \bar{n}_{B}+k_{A C} \bar{n}_{C}
$$

together with

$$
\bar{n}_{A}+\bar{n}_{B}+\bar{n}_{C}=n_{A}+n_{B}+n_{C}=n
$$

expressing the conservation of the total amount. If the equilibrium ratios $\bar{n}_{A}: \bar{n}_{B}: \bar{n}_{C}$ are known this implies two independent relations between the 6 coefficients $k_{A B}, k_{A C}, \cdots k_{C B}$, leaving 4 of them free.

Here, however, the chemists are accustomed to impose a very interesting additional restriction, namely: when the equilibrium is reached each indi- 
viual reaction must balance itself. They require that the transition $A \rightarrow B$ must take place just as frequently as the reverse transition $B \rightarrow A$ etc. Now if the ratios between $\bar{n}_{A}, \bar{n}_{B}$, and $\bar{n}_{C}$ are known the condition of detailed balancing imposes three relations between the $k$ 's instead of the two expressed by (3.3), namely

$$
\begin{aligned}
& k_{B A} \bar{n}_{A}=k_{A B} \bar{n}_{B} \\
& k_{C B} \bar{n}_{B}=k_{B C} \bar{n}_{C} \\
& k_{A C} \bar{n}_{C}=k_{C A} \bar{n}_{A} .
\end{aligned}
$$

These reciprocal relations are analogous to (2.4). From (3.5) we can obtain just one relation between $k_{A B}$ etc. alone, namely

$$
k_{A C} k_{C B} k_{B A}=k_{A B} k_{B C} k_{C A} \text {. }
$$

This relation is not necessary for fulfilling the thermodynamic requirements; those are satisfied as soon as an equilibrium exists, and the existence of an equilibrium is secured by any set of positive values of $k_{A B} \cdots k_{C B}$. In terms of equations: (3.5) is not contained in (3.3).

Suppose that (3.5) were not fulfilled, how could the equilibrium be maintained? Besides a certain number of transitions balancing each other directly according to the scheme

$$
\begin{aligned}
& A \rightleftarrows B \\
& B \rightleftarrows C \\
& C \rightleftarrows A
\end{aligned}
$$

we should have additional transitions taking place around the cycle

$$
\underset{C \rightarrow B}{A}
$$

Now the idea of an equilibrium maintained by a mechanism like (3.6) whether entirely or only in part, is not in harmony with our notion that molecular mechanics has much in common with the mechanics of ordinary conservative dynamical systems. Barring certain exceptional cases $^{8}$ which can readily be recognized and sorted out, the dynamical laws of familiar conservative systems are always reversible, that means: if the velocities of all the particles present are reversed simultaneously the particles will retrace their former paths, reversing the entire succession of configurations. We like to think that the dynamical laws which govern the world of atoms are also reversible. The information that we have about the atoms affords considerable support for this belief of ours, and we have no serious counter-indications, if any. If the dynamical laws of an isolated molecular system are reversible the kinetic theory requires that in the long run every type of motion must occur just as often as its reverse, because the congruence of the two types of motion makes them apriori equivalent. This implies that if we wait a long time so as to make sure of thermodynamic equilibrium, in the end every type of motion

- Coriolis forces, external magnetic fields (and permanent magnets). See $\$ 7$. 
is just as likely to occur as its reverse. One consequence of this principle of dynamical reversibility is the condition that when a molecule changes a certain number of times per second from the configuration $A$ to the configuration $B$ the direct reverse transition $B \rightarrow A$ must take place equally of ten, as expressed by (3.5).

In order to see the full analogy between the reciprocal relations (3.5) and 2.4) we must find an expression for the change in free energy involved by the chemical reactions (3.1). Assuming in accordance with the ideal mass-action formula (3.2) that the molecules $A, B$, and $C$ form an ideal solution we may express the dependance of the "free energy at constant pressure" $Z$ of $n_{A}, n_{B}$ and $n_{C}$ in the form

Thus

$$
Z=Z_{\text {min }}+R T\left(n_{A} \log \left(n_{A} / \bar{n}_{A}\right)+n_{B} \log \left(n_{B} / \bar{n}_{B}\right)+n_{C} \log \left(n_{C} / \bar{n}_{C}\right)\right)(3.7)
$$

$$
\delta Z_{(P, T, n)}=R T\left(\log \left(n_{A} / \bar{n}_{A}\right) \delta n_{A}+\log \left(n_{B} / \bar{n}_{B}\right) \delta n_{B}+\log \left(n_{C} / \bar{n}_{C}\right) \delta n_{C}\right)
$$

We shall introduce the notation

and write

$$
x_{A}=n_{A}-\bar{n}_{A} \quad \text { etc. }
$$

$$
\delta Z=-X_{A} \delta x_{A}-X_{B} \delta x_{B}-X_{C} \delta x_{C} .
$$

In order to obtain proportionality between the "forces" $X$ and the displacements $x$ we must limit ourselves to the consideration of cases where the system is nearly in equilibrium, i.e.

Then we have

$$
x_{A}<<\bar{n}_{A} \text { etc. }
$$

$$
\begin{aligned}
X_{A} & =-R T \log \left(n_{A} / \bar{n}_{A}\right) \sim-\frac{R T}{\bar{n}_{A}} x_{A} \\
X_{B} & \sim-\frac{R T}{\bar{n}_{B}} x_{B} \\
X_{C} & \sim-\frac{R T}{\bar{n}_{C}} x_{C} .
\end{aligned}
$$

Observing (3.3) and 3.8) the Eqs. (3.2) may be written in form

$$
\dot{x}_{A}=\frac{d x_{A}}{d t}=-\left(k_{B A}+k_{C A}\right) x_{A}+k_{A B} x_{A B}+k_{A C} x_{C}
$$

or, finally, with the aid of (3.9)

$$
\begin{aligned}
& \dot{x}_{A}=\left(k_{B A}+k_{C A}\right) \frac{\bar{n}_{A}}{R T} X_{A}-\frac{k_{A B} \bar{n}_{B}}{R T} X_{B}-\frac{k_{A C} \bar{n}_{C}}{R T} X_{C} \\
& \dot{x}_{B}=-\frac{k_{B A} \bar{n}_{A}}{R T} X_{A}+\left(k_{A B}+k_{C B}\right) \frac{\bar{n}_{B}}{R T} X_{B}-\frac{k_{B C} \bar{n}_{C}}{R T} X_{C} \\
& \dot{x}_{C}=-\frac{k_{C A} \bar{n}_{A}}{R T} X_{A}-\frac{k_{C B} \bar{n}_{B}}{R T} X_{B}+\left(k_{A C}+k_{B C}\right) \frac{\bar{n}_{C}}{R T} X_{C} .
\end{aligned}
$$


Comparing (3.10) with (2.3) the complete analogy between (3.5) and (2.4) is apparent. ${ }^{9}$

This comparison suggests that reciprocal relations of the type (2.4) can be derived from the principle of microscopic reversibility. It is true that in the above derivation of (3.5) we started out from a special picture; but we saw at least that this picture was not by itself sufficient to yield the reciprocal relations (3.5). The feature that simplified the consideration so much was the assumed mechanism of elementary transitions, which permitted us to apply the condition of reversibility, or "detailed balancing," to each type of transitions separately. We can already recognize the essential elements in the derivation of (1.2) (which is only another form of (2.4)) from the electron theory of metals. In those deductions it was assumed that the rates of transport processes were limited by collisions between particles whose velocities were distributed according to Maxwell's law. Now the collision is in effect a kind of transition leading from a state characterized by one pair of velocities $\left(v_{1}{ }^{\prime}, v_{2}{ }^{\prime}\right)$ to another state $\left(v_{1}{ }^{\prime \prime}, v_{2}{ }^{\prime \prime}\right)$. The requirement of microscopic reversibility enters through the condition that the transitions:

$$
\left(\boldsymbol{v}_{1}{ }^{\prime}, v_{2}{ }^{\prime}\right) \rightarrow\left({v_{1}}^{\prime \prime}, v_{2}{ }^{\prime \prime}\right) \text { and }\left(-v_{1}{ }^{\prime \prime},-v_{2}{ }^{\prime \prime}\right) \rightarrow\left(-v_{1}{ }^{\prime},-v_{2}{ }^{\prime}\right)
$$

must occur equally of ten when the system has reached thermodynamic equilibrium.

For a general derivation of reciprocal relations like (2.4) we should like to make no reference whatsoever to any particular type of mechanism. We understand already that any mechanism representing an irreversible process as the net resultant of many independent elementary transitions is liable to yield the expected relations; but we have to deal with many cases where no such mechanism can reasonably be assumed. For that reason we want to consider only the integral changes that are involved by the irreversible process. At the same time we want to apply our basic assumption of microscopic reversibility. There is just one possible way: We must consider the fluctuations in a system which has been left isolated for a length of time that is normally sufficient to secure thermodynamic equilibrium.

\section{Heat Conduction and Fluctuations of the Distribution of EnERgy in a Crystal}

We shall show in a simple concrete example how the principle of microscopic reversibility demands reciprocal relations in transport processes. The

${ }^{9}$ Strictly speaking, one may object that there is a difference between the two cases inasmuch as $x_{A}, x_{B}$, and $x_{C}$ are subject to the restriction:

$$
\begin{aligned}
& x_{A}+x_{B}+x_{C}=0 \\
& \dot{x}_{A}+\dot{x}_{B}+\dot{x}_{C}=0
\end{aligned}
$$

(because of (3.3) and (3.8)); so that (3.10) actually contains only two independent variables. However, this only means that (3.10) is analogous to a set of equations of the type (2.3) written out for two variables $J_{1}, J_{2}$ instead of three, i.e., (2.1), with only one reciprocal relation (2.2). This statement may be verified by eliminating one of the variables $x$, say $x_{C}$, in the equations (3.7) etc. The whole complication is quite trivial, and we shall spare ourselves the trouble of going through the details of the calculation,here. 
consideration of fluctuations will enable us to treat the stationary thermodynamic equilibrium as the average result of transitions in different directions without any explicit assumption regarding elementary transitions. We must of course connect the fluctuations with the macroscopic laws of thermodynamics and irreversible processes by reasonable general assumptions. The principles involved are not new; they are classical theorems of statistical mechanics. To begin with, let us contrast the thermodynamic to the statistical point of view.

In thermodynamics we assume that when a system has been left isolated for a sufficient length of time it will reach a state of equilibrium, where all the visible properties of the system remain constant. The approach to equilibrium is irreversible.

The kinetic theory allows only a statistical interpretation of the second law of thermodynamics; the reversible fundamental laws of dynamics are not compatible with absolutely irreversible processes. For instance, we take the point of view that the uniform distribution of a gas within an enclosure is a stable equilibrium state simply because any other distribution is much less probable. We recognize the possibility that a gas may by some freak occurrence compress itself without external aid into one-half of the available volume, although we do not expect ever to observe such an event; the probability is exceedingly small, namely: $2^{-N}$, where $N$ is the total number of molecules present. While such large deviations from tne normal are exceedingly rare smaller deviations will occur much more frequently, and the common deviations from the average number of molecules in either half of the vessel will be of the order of magnitude $(N)^{\frac{1}{2}}$.

In connection with the theory of heat conduction we shall naturally study the fluctuating deviations from the thermal equilibrium. For instance, we may follow the variations in the position of the center of gravity of the energy. ${ }^{10}$ According to thermodynamics this point will remain at rest after thermal equilibrium has been reached. According to statistical mechanics we may expect a succession of slightly asymmetrical distributions of energy. Then the motion of the center of gravity of the distribution is a legitimate object of study.

The ordinary macroscopic laws of heat conduction relate the flow of heat to the temperature gradient at any given time, so that the distribution of energy at any one time determines all subsequent distributions. From the point of view of the kinetic theory this pre-determination cannot be interpreted as absolute; for instance, we do not expect to find an ultimate motionless and completely uniform distribution as required by the macroscopic laws. Still these laws will have a quite definite meaning in the kinetic theory as well. Suppose that we reproduce the same distribution of energy many times independently. Then the subsequent distributions will not be exactly the same in all cases, nor will the rates of change agree exactly with the macroscopic laws in each individual case; but the average rates for a large number

${ }^{10}$ Coordinates $\xi_{1}=\alpha_{1} / E ; \xi_{2}=\alpha_{2} / E, \alpha_{1}$ and $\alpha_{2}$ being defined by (4.3). We must of course make an arbitrary convention about the total content of energy $E$. 
of cases will follow definite laws, and these laws will agree exactly with the macroscopic laws for the conduction of heat.

We also expect that an asymmetric distribution of energy will be exactly the same whether it has arisen accidentally from fluctuations or has been produced purposely by means of suitable external heating and cooling. Once a given distribution of energy is present its history is immaterial, but, as we have pointed out above, the distribution that is present at any given time determines an average expectancy for the rates of the subsequent changes. In all instances this average expectancy for the rate of transport of heat is related to the momentary distribution by the ordinary macroscopic laws for the conduction of heat.

We shall consider the simplest case that is not trivial, namely: a crystal with a 3-, 4- or 6- counting axis of symmetry $\left(x_{3}\right)$

$$
\begin{aligned}
x_{1}+i x_{2} \rightarrow\left(x_{1}+i x_{2}\right) e^{2 x i m / n} & (n=3,4 \text { or } 6) \\
& (m=1,2 \cdots)
\end{aligned}
$$

and no other elements of symmetry except possibly a plane of symmetry perpendicular to $x_{3}\left(x_{3} \rightarrow-x_{3}\right)$ and a center of symmetry $\left(x_{1}, x_{2}, x_{3} \rightarrow-x_{1},-x_{2}\right.$, $\left.-x_{3}\right)$. The equations for the conduction of heat take the form (2.8), or

$$
\begin{aligned}
& -T J_{1}=\lambda_{1} \partial T / d x_{1}+\omega_{3} \partial T / d x_{2} \\
& -T J_{2}=-\omega_{3} \partial T / d x_{1}+\lambda_{1} \partial T / d x_{2} \\
& -T J_{3}=\lambda_{3} \partial T / d x_{3} .
\end{aligned}
$$

Since clockwise and counterclockwise rotations are not equivalent, and $\omega_{3} \geq 0$ and the spiral motion of heat which we mentioned in $\$ 2$ are allowed by the symmetry of the crystal. However, if we may rely on the principle of microscopic reversibility, then a state of motion in any direction is equivalent to a state of motion in the opposite direction, and for that reason $\omega_{3}$ must vanish. Argument and statements are admittedly incomplete. We could not reason in such a simple manner if the case were not so symmetrical, nor would the results be quite as simple. However, let us undertake a more detailed analysis.

We shall study the fluctuations of the distribution of energy in an isolated crystal. In order to take full advantage of the rotational symmetry expressed by (4.1) and (4.2) we give the external shape of the crystal rotational symmetry about the $x_{3}$ axis. The origin of our frame of reference may be at the center of gravity of the crystal; the orientation of the axes is already determined by the symmetry. We shall consider displacements of heat in the directions $x_{1}$ and $x_{2}$. We measure the asymmetry of a given distribution of heat by the moments

$$
\begin{aligned}
& \alpha_{1}=\int \epsilon \cdot x_{1} d V \\
& \alpha_{2}=\int \epsilon \cdot x_{2} d V
\end{aligned}
$$


where $\epsilon=\epsilon\left(x_{1}, x_{2}, x_{3}\right)$ is the local density of energy, ${ }^{11}$ and the integration is extended over the total volume $V$ of the crystal. The displacements $\alpha_{1}$ and $\alpha_{2}$ will differ a little from zero most of the time because of fluctuations; their averages will vanish

$$
\bar{\alpha}_{1}=\bar{\alpha}_{2}=0 .
$$

For reasons of symmetry the average squares of $\alpha_{1}$ and $\alpha_{2}$ must be equal, and displacements in the directions $x_{1}$ and $x_{2}$ will be independent

$$
\overline{\alpha_{1}^{2}}=\overline{\alpha_{2}^{2}} ; \overline{\alpha_{1} \alpha_{2}}=0 \text {. }
$$

The same displacement $\left(\alpha_{1}, \alpha_{2}\right)$ of energy may be produced by different distributions of energy, and, accordingly, of temperature. However, to each value of $\alpha_{1}$, there will correspond a certain average temperature gradient

$$
\overline{\partial T / d x_{1}}{ }^{\alpha_{1}}=-T \bar{X}_{1}\left(\alpha_{1}\right)
$$

in the $x_{1}$ direction, the averaging to be extended over all cases where $\alpha_{1}$ takes the same value, and in each case over all volume elements of the crystal. This average gradient will be proportional to $\alpha_{1}$ :

$$
\overline{\partial T / d x_{1}}{ }^{\alpha_{1}}=-T \bar{X}_{1}\left(\alpha_{1}\right)=T C \alpha_{1} \text {. }
$$

In a similar manner

$$
\overline{\partial T / d x_{2}} \alpha_{2}=-T \bar{X}_{2}\left(\alpha_{2}\right)=T C \alpha_{2}
$$

and if we consider a displacement $\left(\alpha_{1}, \alpha_{2}\right)$ in an arbitrary direction:

$$
\begin{aligned}
& -T \bar{X}_{1}\left(\alpha_{1}, \alpha_{2}\right)=T C \alpha_{1} \\
& -T \bar{X}_{2}\left(\alpha_{1}, \alpha_{2}\right)=T C \alpha_{2}
\end{aligned}
$$

The factor $C$ can be calculated on the basis of the general theory of fluctuations. ${ }^{12}$ In this particular case we shall be content to know that $\alpha_{1}$ causes no temperature gradient in the $x_{2}$ direction, and vice versa, in spite of the fact that rotations in opposite directions in the $x_{1}, x_{2}$ plane are not equivalent. The reason is that in questions regarding the distribution the anisotropy of the crystal is immaterial anyway, and only the external boundary of the crystal is important, because all the volume elements of a homogeneous crystal are equivalent irrespective of location or mutual connections. The anisotropy becomes important as soon as one considers the rate of exchange of energy.

The temperature gradient determines the average rate of transport of heat according to (4.2)

$$
\begin{aligned}
& \bar{J}_{1}\left(\alpha_{1}, \alpha_{2}\right)=\lambda_{1} \bar{X}_{1}\left(\alpha_{1}, \alpha_{2}\right)+\omega_{3} \bar{X}_{2}\left(\alpha_{1}, \alpha_{2}\right)=-\lambda_{1} C \alpha_{1}-\omega_{3} C \alpha_{2} \\
& \bar{J}_{2}\left(\alpha_{1}, \alpha_{2}\right)=-\omega_{3} \bar{X}_{1}\left(\alpha_{1}, \alpha_{2}\right)+\lambda_{1} \bar{X}_{2}\left(\alpha_{1}, \alpha_{2}\right)=\omega_{3} C \alpha_{1}-\lambda_{1} C \alpha_{2} .
\end{aligned}
$$

${ }^{11}$ Strictly speaking, one may identify heat with energy only when the crystal has no thermal expansion in any direction. In general heat must be defined as energy less work of deformation and expansion. This complicates the discussion slightly without changing the results; the symmetry conditions (4.4) and (4.5) remain valid.

${ }^{12}$ Einstein, Ann. d. Physik 33, 1275 (1910). 
Finally, if $V$ is the volume, then $V \bar{J}_{1}$ is the total rate of transport of heat in the $x_{1}$ direction:

$$
d \alpha_{1} / d t=V \bar{J}_{1}
$$

and we obtain

$$
\begin{aligned}
& \overline{d \alpha_{1} / d t}=-\lambda_{1} V C \alpha_{1}-\omega_{3} V C \alpha_{2} \\
& \overline{d \alpha_{2} / d t}=\omega_{3} V C \alpha_{1}-\lambda_{1} V C \alpha_{2} .
\end{aligned}
$$

Suppose now that we watch our crystal for a great length of time. Whenever the displacement of energy in the $x_{1}$ direction happens to be $\alpha_{1}=\alpha_{1}{ }^{\prime}$ we note down the displacement $\alpha_{2}$ in the $x_{2}$ direction $\Delta t$ seconds later. The average of a great number of such observations we shall denote by

$$
\bar{\alpha}_{2}\left(\Delta t, \alpha_{1}^{\prime}\right) \text {. }
$$

For reasons of symmetry

$$
\bar{\alpha}_{2}\left(0, \alpha_{1}{ }^{\prime}\right)=0
$$

because this average does not depend on the rate of transport, but only on the relative probabilities of different distributions of energy (simultaneous values of $\alpha_{1}$ and $\alpha_{2}$ ). The average change of $\alpha_{2}$ in a time $\Delta t$ subsequent to distributions for which $\alpha_{1}=\alpha_{1}{ }^{\prime}$ is therefore

$$
\bar{\alpha}_{2}\left(\Delta t, \alpha_{1}{ }^{\prime}\right)-\bar{\alpha}_{2}\left(0, \alpha_{1}{ }^{\prime}\right)=\bar{\alpha}_{2}\left(\Delta t, \alpha_{1}{ }^{\prime}\right) .
$$

If $\Delta t$ is sufficiently small we can calculate this change from (4.7):

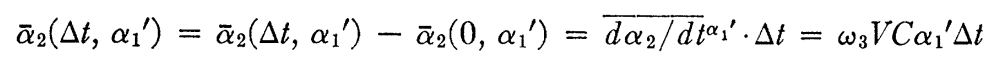

Now we can calculate the average product

$$
\overline{\alpha_{1}(t) \alpha_{2}(t+\Delta t)}=\lim _{t^{\prime \prime} \rightarrow \infty} \frac{1}{t^{\prime \prime}-t^{\prime}} \int_{t=t^{\prime}}^{t=t^{\prime \prime}} \alpha_{1}(t) \alpha_{2}(t+\Delta t) d t
$$

Obviously:

$$
\overline{\alpha_{1}(t)} \overline{\alpha_{2}(t+\Delta t)}=\overline{\alpha_{1}^{\prime} \bar{\alpha}_{2}\left(\Delta t, \alpha_{1}{ }^{\prime}\right)}=\omega_{3} V C \overline{\alpha_{1}{ }^{2}} \Delta t \text {. }
$$

In a similar manner, or directly from (4.9a) by taking into account the rotational symmetry (4.1) of the crystal we obtain:

$$
\overline{\alpha_{2}(t) \alpha_{1}(t+\Delta t)}=-\omega_{3} V C \overline{\alpha_{1}^{2}} \Delta t=-\omega_{3} V C \overline{\alpha_{2}^{2}} \Delta t .
$$

Here

$$
\overline{\alpha_{1}^{2}}=\overline{\alpha_{2}^{2}}
$$

The principle of microscopic reversibility demands that a displacement $\alpha_{1}=\alpha_{1}{ }^{\prime}$ of energy in the $x_{1}$ direction, followed $\tau$ seconds later by a displacement $\alpha_{2}=\alpha_{2}{ }^{\prime \prime}$ in the $x_{2}$ direction, must occur just as of ten as $\alpha_{2}=\alpha_{2}{ }^{\prime \prime}$, followed $\tau$ seconds later by $\alpha_{1}=\alpha_{1}{ }^{\prime}$. Consequently

$$
\overline{\alpha_{1}(t) \alpha_{2}(t+\tau)}=\overline{\alpha_{2}(t) \alpha_{1}(t+\tau)}
$$


where $\tau$ may have any value, for example $\tau=\Delta t$. Comparing with (4.9a, b) we have

$$
\omega_{3}=0
$$

in accordance with Voigt's experimental result, ${ }^{7}$ the simplest example of a reciprocal relation in irreversible processes.

Here we have taken a little more out of the principle of microscopic reversibility than we did in section 3 with the assertion that transitions between two (classes of) configurations $A$ and $B$ should take place equally of ten in the directions $A \rightarrow B$ and $B \rightarrow A$ in a given time $\tau$. Above we have discussed transitions between different distributions of energy. We must expect that the energy will depend not only on the configuration of elementary particles, but also on their velocities; we do not know exactly how. However, assuming microscopic reversibility, the energy must depend on the velocities in such a manner that every type of motion has the same energy as its reverse; otherwise a "reverse" energy would exist, different from the ordinary energy but with similar properties of conservation, and we should not know the energy as a unique function of the state of the system. If the energy can be localized so that we can speak about distributions of energy in space, the distribution of energy must be the same for corresponding phases of direct and reverse motion, or similar discrepancies would arise. Since, by hypothesis, direct and reverse motion of every conceivable type occur pairwise equally of ten, if $A$ and $B$ be two distributions of energy, the transitions $A \rightarrow B$ and $B \rightarrow A$ (in time intervals of a given length $\tau$ ) must occur equally of ten.

We shall comment on another question regarding the premises of the derivation, although the substance of a satisfactory answer is known from a famous discussion between Loschmidt and Boltzmann. ${ }^{13}$ We have assumed microscopic reversibility, and at the same time we have assumed that the average decay of fluctuations will obey the ordinary laws of heat conduction. Already an apparent contradiction occurs when we consider the simpler case of heat conduction in one dimension. Let $\alpha$ be a displacement of heat, then:

$$
\ddot{\alpha}=d \bar{\alpha} / d t=-K \alpha .
$$

Microscopic reversibility requires

$$
\bar{\alpha}\left(\tau, \alpha^{\prime}\right)=\bar{\alpha}\left(-\tau, \alpha^{\prime}\right) .
$$

Clearly

$$
\dot{\alpha}\left(\tau, \alpha^{\prime}\right)=-\dot{\alpha}\left(-\tau, \alpha^{\prime}\right)
$$

and :

$$
\ddot{\alpha}\left(0, \alpha^{\prime}\right)=-\dot{\alpha}\left(0, \alpha^{\prime}\right)=0 .
$$

According to the ordinary laws for conduction of heat $\bar{\alpha}$ decreases for positive $\tau$ (if $\alpha^{\prime}>0$ ). According to (4.13), then, $\bar{\alpha}$ increases for negative $\tau$ (average growth of fluctuations), and $\ddot{\alpha}=0$ for $\tau=0$. It may appear somewhat startling

${ }^{13}$ See P. and T. Ehrenfest. F.n7. d math. Wiss. IV. 32. 
that we apply (4.12) to fluctuations only for $\tau>0$, and not for $\tau \leqq 0$. Yet in this there is no logical contradiction - we have stated bluntly and honestly that $\dot{\alpha}$ has a discontinuity for $\tau=0$-but such a statement disappoints our expectation of continuity in nature. However, the objection is removed when we recognize that (4.12) is only an approximate description of the process of conduction, neglecting the time needed for acceleration of the heat flow. This time $\tau_{0}$ is probably rather small, e.g. in gases it ought to be of the same order of magnitude as the average time spent by a molecule between two collisions. For practical purposes the time-lag can be neglected in all cases of heat conduction that are likely to be studied, and this approximation is always involved in the formulation of laws like (4.12), (4.7) and (4.2). Even the differential form (e.g. (4.8)) of these equations is justified; because we can usually choose a time $\Delta t$ such that:

$$
1 \gg K \Delta t \gg K \tau_{0} .
$$

Then following $t=\tau_{0}$, which is practically the same as $t=0$, we have a time interval $\Delta t \gg \tau_{0}$ in which (by (4.12)) $\alpha$ and therefore $d \alpha / d t$ are sensibly constant. We may also recall that the time needed for equalization of temperature in a body is proportional to the square of its linear dimensions $l$, i.e.:

$$
K \sim 1 / l^{2} .
$$

In gases $K \tau_{0}$ should be of the order $l^{2} / \Lambda^{2}$, where $\Lambda$ is the mean free path. The ordinary laws for conduction of heat are therefore asymptotic laws for $l \gg \Lambda$.

The preceding considerations leading to (4.11) are easily extended to the more general case of heat conduction in a crystal of arbitrary symmetry. The phenomenological equations have the form

$$
\begin{aligned}
& J_{1}=L_{11} X_{1}+L_{12} X_{2}+L_{13} X_{3} \\
& J_{2}=L_{21} X_{1}+L_{22} X_{2}+L_{23} X_{3} \\
& J_{3}=L_{31} X_{1}+L_{32} X_{2}+L_{33} X_{3}
\end{aligned}
$$

where

$$
T X_{1}=-\partial T / d x_{1} ; T X_{2}=-\partial T / d x_{2} ; T X_{3}=-\partial T / d x_{3} .
$$

We shall derive the reciprocal relations:

$$
L_{12}=L_{21} ; L_{23}=L_{32} ; L_{31}=L_{13} .
$$

We chose the external shape of our crystal spherical. Since the anisotropy of the crystal has nothing to do with the distribution of heat the arguments leading to (4.4) and (4.5) apply equally well in this case, and we obtain

$$
\begin{aligned}
\overline{\alpha_{1}^{2}}=\overline{\alpha_{2}^{2}} & =\overline{\alpha_{3}^{2}} ; \overline{\alpha_{1} \alpha_{2}}=\overline{\alpha_{2} \alpha_{3}}=\overline{\alpha_{3} \alpha_{1}}=0 \\
& -\bar{X}_{1}\left(\alpha_{1}, \alpha_{2}, \alpha_{3}\right)=C \alpha_{1} \\
& -\bar{X}_{2}\left(\alpha_{1}, \alpha_{2}, \alpha_{3}\right)=C \alpha_{2} \\
& -\bar{X}_{3}\left(\alpha_{1}, \alpha_{2}, \alpha_{3}\right)=C \alpha_{3} .
\end{aligned}
$$


Instead of (4.6) and (4.7) we find

$$
\begin{aligned}
\overline{J_{1}}\left(\alpha_{1}, \alpha_{2}, \alpha_{3}\right) & =-L_{11} C \alpha_{1}-L_{12} C \alpha_{2}-L_{13} C \alpha_{3}, \text { etc. } \\
\overline{d \alpha_{1} / d t} & =-L_{11} V C \alpha_{1}-L_{12} V C \alpha_{2}-L_{13} V C \alpha_{3}, \text { etc. }
\end{aligned}
$$

The analogy of (4.8) becomes

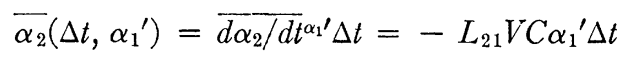

and we have in the place of (4.9)

$$
\overline{\alpha_{1}(t)} \overline{\alpha_{2}(t+\Delta t)}=\overline{\alpha_{1}^{\prime} \bar{\alpha}_{2}\left(\Delta t, \alpha_{1}{ }^{\prime}\right)}=-L_{21} V C \overline{\alpha_{1}{ }^{2}} \Delta t
$$

Likewise

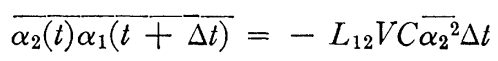

Microscopic reversibility (4.10) requires

$$
\overline{\alpha_{1}(t) \alpha_{2}(t+\overline{\Delta t)}}=\overline{\alpha_{2}(t) \alpha_{1}(t+\Delta t)} .
$$

Comparison with $(4.16 \mathrm{a}, \mathrm{b})$ and $(4.15)$ yields the generalization of $(4.11)$

$$
L_{12}=L_{21} \text {. }
$$

The other two equations in (2.4) are obviously derived in the same way.

\section{The Principle of the Least Dissipation of Energy}

Like the reciprocal relations of mechanics and thermodynamics the relations (2.4) can be expressed in terms of a potential, and permit the formulation of a variation principle. As a preliminary we re-write (2.3) and (4.14) expressing the "forces" $X$ by the "velocities" $J$ :

$$
\begin{aligned}
& -\frac{1}{T} \frac{\partial T}{d x_{1}}=X_{1}=R_{11} J_{1}+R_{12} J_{2}+R_{13} J_{3} \\
& -\frac{1}{T} \frac{\partial T}{d x_{2}}=X_{2}=R_{21} J_{1}+R_{22} J_{2}+R_{23} J_{3} \\
& -\frac{1}{T} \frac{\partial T}{d x_{3}}=X_{3}=R_{31} J_{1}+R_{32} J_{2}+R_{33} J_{3}
\end{aligned}
$$

where $R_{11} \cdots R_{33}$ are connected with $L_{11} \cdots L_{33}$ in (2.3) by the well known relations

$$
\sum_{m=1}^{3} L_{i m} R_{m k}=\sum_{m=1}^{3} R_{i m} L_{m k}=\delta_{i k}= \begin{cases}1 & (i=k) \\ 0 & (i \gtrless k)\end{cases}
$$

and the reciprocal relations (2.4) are equivalent to:

$$
R_{12}=R_{21} ; R_{13}=R_{31} ; R_{23}=R_{32} .
$$

Here, if we write:

$$
2 \phi(J, J) \equiv \frac{1}{T} \sum_{i, k} R_{i k} J_{i} J_{k}
$$


the relations (5.1) may be written:

$$
\frac{1}{T} X_{k}=\frac{\partial}{d x_{k}}\left(\frac{1}{T}\right)=\frac{\partial \phi(J, J)}{d J_{k}} .
$$

We also observe that:

$$
2 T \phi(J, J) \equiv \sum_{k=1}^{3} J_{k} T \partial \phi / d J_{k}=J_{1} X_{1}+J_{2} X_{2}+J_{3} X_{3}
$$

The function $\phi(J, J)$ we shall call the dissipation-function. It is a direct generalization of a function which was introduced by Lord Rayleigh ${ }^{14}$ and applied to mutual interaction of frictional forces; it plays the part of a potential for such forces. Actually Lord Rayleigh used the function $F(J, J)$ $=T \phi(J, J)$ and called $F$ the dissipation-function; for our purposes we shall find the function $\phi$ more generally useful. As we shall see immediately, $2 \phi(J$, $J$ ) equals the rate of production of entropy due to heat flow across a volume element (of unit size), so that $2 T \phi=2 F$ equals the rate of "dissipation" of free energy.

The rate of local accumulation of heat equals

$$
T d s / d t=-\operatorname{div} J=-\partial J_{1} / d x_{1}-\partial J_{2} / d x_{2}-\partial J_{3} / d x_{3}
$$

writing $s$ for the local entropy density, and the total rate of increase of the entropy $S$ equals

$$
d S / d t=\int(d s / d t) d V=\int\left(-\frac{1}{T} \operatorname{div} J\right) d V .
$$

By Green's theorem:

$$
\begin{aligned}
& \iiint(-\operatorname{div} J) \frac{1}{T} d V+\iint \frac{J_{n}}{T} d \Omega=\iiint\left(J, \operatorname{grad} \frac{1}{T}\right) d V \\
= & \iiint\left(J_{1} \frac{\partial}{d x_{1}}\left(\frac{1}{T}\right)+J_{2} \frac{\partial}{d x_{2}}\left(\frac{1}{T}\right)+J_{3} \frac{\partial}{d x_{3}}\left(\frac{1}{T}\right)\right) d V
\end{aligned}
$$

where the double integral on the left is extended over the boundary $\Omega$ of the body in question, and $J_{n}$ is the normal component of the heat flow at the boundary. If we write

$$
\dot{S}^{*}\left(J_{n}\right) \equiv \iint\left(J_{n} / T\right) d \Omega
$$

for the entropy given off to the surroundings, and

$$
\dot{S}(J) \equiv \iiint\left(-\frac{1}{T} \operatorname{div} J\right) d V
$$

${ }^{14}$ Lord Rayleigh, Proc. Math. Soc. London 4, 357, [363], (1873). Theory of Sound, (London, MacMillan Co., 1st ed. 1877), Vol. I, p. 78; (2d ed. 1894), Vol. I, p. 102. 
for the entropy change of the system proper, we have:

$$
\begin{aligned}
\dot{S}(J)+\dot{S}^{*}\left(J_{n}\right) & \equiv \int\left(J, \operatorname{grad} \frac{1}{T}\right) d V \equiv \int \sum_{k} J_{k} \frac{\partial}{d x_{k}}\left(\frac{1}{T}\right) d V \\
& \equiv \int \frac{1}{T} \sum_{k} J_{k} X_{k} d V
\end{aligned}
$$

(observing (4.14)). Now, by (5.4) and (5.5)

$$
\int \sum_{k} J_{k} \frac{\partial}{d x_{k}}\left(\frac{1}{T}\right) d V=\int \sum_{k} J_{k} \frac{\partial \phi(J, J)}{d J_{k}} d V=\int 2 \phi(J, J) d V .
$$

Inserting this in (5.9) we find

$$
2 \Phi(J, J) \equiv 2 \int \phi(J, J) d V=\dot{S}(J)+\dot{S}^{*}\left(J_{n}\right)
$$

Now we shall show that the relations (5.4) are equivalent to the variation principle

$$
\dot{S}(J)+\dot{S}^{*}\left(J_{n}\right)-\Phi(J, J)=\text { maximum }
$$

with the conventions that the temperature distribution $T\left(x_{1}, x_{2}, x_{3}\right)$ is prescribed, the flow $J\left(x_{1}, x_{2}, x_{3}\right)$ is varied, and the functions $\dot{S}, \dot{S}^{*}$ and $\Phi$ are defined by (5.7), (5.8) and (5.10), respectively (reading ( $\equiv)$, but not (=): "identical in $\left.J^{\prime \prime}\right)$. Observing (5.9) we have

$$
\begin{aligned}
& \delta\left[\dot{S}(J)+\dot{S}^{*}\left(J_{n}\right)-\Phi(J, J)\right] \\
& =\delta \int\left[\sum_{k} J_{k} \frac{\partial}{d x_{k}}\left(\frac{1}{T}\right)-\phi(J, J)\right] d V \\
& =\int \sum_{k}\left[\frac{\partial}{d x_{k}}\left(\frac{1}{T}\right)-\frac{\partial}{d J_{k}} \phi(J, J)\right] \delta J_{k} d V
\end{aligned}
$$

so that (5.4) is clearly equivalent to

$$
\delta\left[\dot{S}(J)+\dot{S}^{*}\left(J_{n}\right)-\Phi(J, J)\right]=0 .
$$

Here, since $\dot{S}$ and $\dot{S}^{*}$ are linear functionals of $J$, and $\Phi$ is a homogeneous quadratic functional, the expression in the brackets can have only one extremum. This extremum is a maximum because $\Phi(J, J)$ must be positivedefinite (otherwise (5.10) would not agree with the second law of thermodynamics).

If the boundary is isolated the restriction

$$
J_{n}=0
$$


enters, and, since then $\dot{S}^{*}\left(J_{n}\right)$ vanishes

$$
\dot{S}(J)-\Phi(J, J)=\text { maximum } .
$$

Thus the vector field $J$ of the heat flow is described by the condition that the rate of increase of the entropy, less the dissipation-function, be a maximum.

In applications the difference between the formulations (5.11) and (5.13) is trivial. From a fundamental point of view (5.13) has some merit of greater simplicity because it applies to an isolated system, and is thus more directly connected with the theory of fluctuations. Above we have demonstrated (5.13) for anisotropic heat conduction. A more general theorem applying to all transport processes (conduction of electricity and heat, and diffusion) can be derived in a similar manner, only it is then necessary to make full use of the general theory of fluctuations, involving Boltzmann's classical relation between entropy $S$ and probability $W$

$$
S=k \log W+\text { const. }
$$

This general development will be deferred to a following communication.

\section{Stationary Flow and Quasi-Thermodynamics}

A brief discussion of the conditions for stationary flow of heat through an anisotropic body will bring out an interesting simple consequence of (5.11) and throw light on the connection with previous quasi-thermodynamic derivations of reciprocal relations in irreversible processes.

The condition for stationary flow of heat is

$$
\operatorname{div} J=\partial J_{1} / d x_{1}+\partial J_{2} / d x_{2}+\partial J_{3} / d x_{3}=0
$$

for the interior of the body under consideration. No heat is accumulated in the interior, so that $\dot{S}(J)=0$. Thus (5.11) becomes:

$$
\dot{S}^{*}\left(J_{n}\right)-\Phi(J, J)=\text { maximum }
$$

Eqs. (6.1) and (6.2) determine the field of flow $J$ as well as the temperatures in the interior when the temperatures at the boundary are prescribed. (An eventual dependence of $\phi(J, J)$ on the temperature should be ignored in carrying out the variation: $\left.\delta \phi(J, J)=\Sigma\left(\partial \phi / d J_{k}\right) \delta J_{k}\right)$. In this case, where $\dot{S}(J)=0,(5.10)$ becomes

$$
\dot{S}^{*}\left(J_{n}\right)=2 \Phi(J, J)
$$

and we have

$$
2\left(\dot{S}^{*}\left(J_{n}\right)-\Phi(J, J)\right)=\dot{S}^{*}\left(J_{n}\right) .
$$

We may therefore state (6.2) in the alternative form

$$
\dot{S}^{*}\left(J_{n}\right)=\text { maximum }
$$

with the restrictions (6.1) and (6.3). 
Now let us consider the effect of obstacles to the heat flow, like cracks in the crystal, introducing restrictions of the type:

$$
J_{n}{ }^{\prime}=0
$$

for the changed heat flow $J^{\prime}$ at certain interior surfaces. Since the field $J$ of the original heat flow made $\dot{S}^{*}\left(J_{n}\right)$ a maximum under restrictions that are also imposed on $J^{\prime}$, we must have

$$
\dot{S}^{*}\left(J_{n}\right) \geqq \dot{S}^{*}\left(J_{n}{ }^{\prime}\right)
$$

Restrictions can only decrease the rate of production of entropy, or cause no change.

Assumptions of the type (6.5) are involved in all quasi-thermodynamic derivations proposed by earlier writers for reciprocal relations in irreversible processes. As good an example as any is Thomson's case (1.1), where $J_{1}$ is the electrical current and $J_{2}$ the heat flow (in the same direction). We maintain a constant temperature gradient $-T X_{2}$, while $X_{1}$ may be varied. The restriction: $J_{1}=0$ may be imposed by breaking the electric circuit. The rate of production of entropy equals

$$
\dot{S}(J)+\dot{S}^{*}\left(J_{n}\right)=(V / T)\left(X_{1} J_{1}+X_{2} J_{2}\right)
$$

(cf. (5.9)). With the aid of (1.1) we transform this relation into:

$T\left(\dot{S}(J)+\dot{S}^{*}\left(J_{n}\right)\right)=\left(V / R_{22}\right)\left[X_{2}^{2}+\left(R_{12}-R_{21}\right) X_{2} J_{1}+\left(R_{11} R_{22}-R_{12} R_{21}\right) J_{1}^{2}\right]$.

Here, if we assume that the restriction

$$
J_{1}=0
$$

makes $\dot{S}+\dot{S}^{*}$ a minimum for given $X_{2}$ (cf. (6.5)), we find

$$
R_{12}-R_{21}=0 \text {. }
$$

In conclusion, let us describe the case which has given name to the "principle of the least dissipation of energy." The flow of heat $J_{n}$ across all sections of the boundary $\Omega$ is prescribed, and the condition

$$
\int J_{n} d \Omega=0
$$

is fulfilled. Then $\dot{S}^{*}\left(J_{n}\right)$ in (6.2) is prescribed, and the condition for stationary flow reduces to

$$
\Phi(J, J)=\text { minimum }
$$

subject to the restrictions: 


$$
\begin{gathered}
J_{n} \text { prescribed } \\
\operatorname{div} J=0 .
\end{gathered}
$$

These conditions determine $J$, and the temperatures are determined everywhere if known at one point.

\section{Non-Reversible Systems}

A dynamical system is reversible as long as the (mechanical) forces dèpend only on the coordinates or, if they depend on the velocities as well, are even functions of these. We know conservative systems which do not fulfill this condition. (i) An electric charge moving in a magnetic field is deflected by a force proportional to the product of charge and velocity. (ii) Relative to a rotating frame of reference a free particle moves as if it were subject to a transverse force proportional to the product of mass and velocity (Coriolis force) besides the centrifugal force.

When magnetic forces and Coriolis forces destroy the reversibility of macroscopic motion we must expect that the microscopic motion will fare no better. The reciprocal relations (2.4) and their equivalent, the principle of the least dissipation of energy (5.11) are derived from the assumption of microscopic reversibility. We may expect that these relations will break down in cases where magnetic or Coriolis forces are acting, and they do. The influence of Coriolis forces on heat conduction is presumably small and not easily studied; but magnetic fields are known to modify the relation between heat flow and temperature gradient in metals. In an isotropic body, the simplest case, the temperature gradient has the same direction as the heat flow as long as no magnetic field is present. However, if a transverse magnetic field is applied the temperature gradient will have a component in the third direction perpendicular to flow and field. The direction of the temperature gradient is rotated with respect to the heat flow, about an axis parallel to the magnetic field. This phenomenon is known as the Righi-Leduc effect. If a circular metal plate is placed perpendicular to the magnetic field, heated in the middle and cooled at the edge, the heat will flow outward in spirals. The equations of the heat flow take the form (2.8), or rather

$$
\begin{aligned}
& J_{1}=\lambda X_{1}+\omega X_{2} \\
& J_{2}=-\omega X_{1}+\lambda X_{2} \\
& J_{3}=\lambda X_{3}
\end{aligned}
$$

where $\omega$ is proportional to the intensity of the magnetic field (for weak fields); the field is thought parallel to the $x_{3}$ direction. The principle of the least dissipation of energy is no longer valid; radial cracks in the plate will increase the rate of radial transport of heat for a given temperature gradient.

More familiar than the Righi-Leduc effect is perhaps the Hall effect. When a constant current is flowing through a metallic conductor a transverse magnetic field causes an e.m.f. perpendicular to both. Eqs. (7.1) describe the isotropic case if $J_{1}, J_{2}, J_{3}$, denote the components of the current and $X_{1}, X_{2}$, $X_{3}$ the components of the electric field. 
In the presence of a magnetic field the principle of microscopic reversibility may be applied in a modified form: The entire motion may be reversed by reversing the magnetic field together with the velocities of all the particles composing a dynamical system. Eqs. (7.1) are in accord with this requirement.

An analogous effect of Coriolis forces is known in hydrodynamics. The principle of the least dissipation of energy applies to the motion of very viscous fluids (Stokes' limiting case) as long as the motion is not referred to a rotating frame of reference. 